\title{
Jamming transitions in amorphous packings of frictionless spheres occur over a continuous range of volume fractions
}

\author{
Pinaki Chaudhuri, ${ }^{1}$ Ludovic Berthier, ${ }^{2}$ and Srikanth Sastry ${ }^{3}$ \\ ${ }^{1}$ Laboratoire PMCN, Université Lyon 1, Université de Lyon, UMR CNRS 5586, 69622 Villeurbanne, France \\ ${ }^{2}$ Laboratoire des Colloïdes, Verres et Nanomatériaux, \\ UMR CNRS 5587, Université Montpellier 2, 34095 Montpellier, France \\ ${ }^{3}$ Jawaharlal Nehru Centre for Advanced Scientific Research, Jakkur Campus, Bangalore 560064, India
}

(Dated: October 30, 2018)

\begin{abstract}
We numerically produce fully amorphous assemblies of frictionless spheres in three dimensions and study the jamming transition these packings undergo at large volume fractions. We specify four protocols yielding a critical value for the jamming volume fraction which is sharply defined in the limit of large system size, but is different for each protocol. Thus, we directly establish the existence of a continuous range of volume fraction where nonequilibrium jamming transitions occur. However, these jamming transitions share the same critical behaviour. Our results suggest that, even in the absence of partial crystalline ordering, a unique location of a random close packing does not exist, and that volume fraction alone is not sufficient to describe the properties of jammed states.
\end{abstract}

PACS numbers: 05.10.-a, 05.20.Jj, 64.70.Pf

Everyday experience shows that it is not possible to compress a disordered assembly of rigid particles beyond a maximal packing fraction. The idea of a critical value for the volume fraction of this 'random close packing' has a long history in science [1]. For the idealized case of hard, spherical, frictionless particles the idea recently emerged that a critical value of the volume fraction, named $\phi_{J}$, can be appropriately defined in the thermodynamic limit [2, 3], and that it corresponds to a critical point ('point $J$ ') with remarkable scaling properties observed when $\phi_{J}$ is approached from either side [2, 4]. A unique critical packing fraction is also found under quasistatic [5] or continuous [6, 7] shearing. Further studies have also established the peculiar properties of configurations prepared exactly at $\phi_{J}$, with specific, singular behaviour of the pair correlation function [8, 9]. This leads to the conjecture that the properties of this critical point can influence the physical behaviour of dense particle systems under various conditions [10].

The identification of a unique critical point for jamming is surprising because two distinct phenomena occur when compressing hard spheres at equilibrium. First, an equilibrated system of monodisperse hard spheres is fully crystalline above $\phi=0.54$. Since crystalline states are efficiently packed, it is in principle always possible to increase the volume fraction of a disordered assembly by increasing the local order [11]. In the studies mentioned above, crystallization is prevented by using size polydispersity which increases the nucleation barrier and can efficiently suppress crystallization at equilibrium. Moreover, these studies employ athermal numerical protocols which do not allow the system to reach equilibrium.

A second relevant phenomenon occurring at equilibrium is the glass transition. Compressing hard spheres at thermal equilibrium, it is found that ergodicity is lost near a volume fraction much lower than $\phi_{J}$, because the relaxation time for structural relaxation becomes larger than experimental timescales [12]. Necessarily, then, the properties of hard sphere glasses should depend on the preparation history, as is well-known for molecular glasses [13]. The resulting interplay between glass and jamming transitions is currently receiving attention, both from theory and simulations. A special density akin to $\phi_{J}$ appears in mean-field glasses undergoing a random first order transition [14]. In this language, $\phi_{J}$ corresponds to the (sharp) appearance of an exponentially large number of metastable states, and such states exist along a continuous range of densities between $\phi_{J}$ and that of the 'glass close packing' 14, 15], where the configurational entropy (or complexity) counting metastable states vanishes. However, none of these mean-field concepts is expected to remain sharply defined in three dimensions [15], and it is important to assess the validity of this interpretation in finite dimensions.

Previous numerical work has shown that different compression rates produce glasses with a pressure which appears to diverge at different densities, at least for finite size systems [16, 17, 18, 19]. Indeed, the analogy 20, 21] of the jamming density with the energy of inherent structures in systems with soft potentials [13], suggests that the jamming densities should depend both on compression rates as well as the initial state from which the glasses are generated. Recently, a compression protocol was specifically devised to prevent any ordering, thus allowing to focus directly on the sole influence of glassy behaviour on jamming [22]. Starting from a wellequilibrated fluid configuration at a given volume fraction, $\phi_{\text {init }}$, one uses a very large compression rate to reach nearly jammed configurations. During these rapid compressions, the glass pressure was observed to diverge within a finite range of volume fractions, even in the thermodynamic limit 22], but the jamming transition was 
not studied.

Since evidence is mounting in favour of both a unique location of the jamming transition with critical properties in some papers, or a continuous range of volume fraction in some other works, it appears timely to reconcile these two lines of research and answer the following important, open questions. Does a continuous range of volume fraction for jamming necessarily result from crystallization or demixing? Can one reconcile the results found using thermal and athermal protocols? Can the remarkable properties of the jamming transition survive if its location is not unique? Here, we provide precise answers to these questions and close the gap between two sets of ideas. We study numerically how amorphous assemblies of hard spheres prepared using the (thermal) tools of Ref. 22] jam at large volume fraction. We then apply the alternative (athermal) tools of Ref. 2] to analyze the properties of jammed configuration at and near the transition. For the specific three-dimensional binary system we use, we can directly establish the existence of a continuous line of $J$-points extending at least in the range $\phi_{J} \in[0.648,0.662]$, all sharing similar critical properties and exponents. Thus, although point $J$ is not unique, its critical properties are.

We study the jamming transition in a threedimensional system of spherical particles using periodic boundary conditions. We use a 50:50 binary mixture of spheres with diameter ratio 1.4. Our preparation protocol combines the fast compression of hard spheres described above 22], to the athermal compression of spheres treated as soft spheres as in Ref. 2]. Specifically, the particles are first treated as hard spheres and equilibrated using Monte-Carlo simulations over a broad range of volume fraction up to $\phi=0.596$. This allows one to obtain disordered configurations representative of the metastable fluid of hard spheres (no crystallisation or demixing is observed for simulations as long as $10^{10}$ Monte-Carlo timesteps). We then use Monte-Carlo simulations to rapidly compress these equilibrated fluid configurations. During these compressions the system has no time to relax and retains a structure very close to the initial fluid states. Thus, contrary to previous work employing slow compressions [18, 19], we are certain that the jammed configurations we produce contain no more order than the original equilibrated fluid configurations.

When (reduced) pressure is very large, $P /\left(\rho k_{B} T\right) \sim$ $10^{3}$, we stop the compression and switch to the athermal procedure of Ref. 2], where spheres are now treated as harmonic spheres interacting through a soft pair potential, $V_{\text {soft }}\left(r_{i j}\right)=\left(1-r_{i j} / \sigma_{i j}\right)^{2}$. Here, $\sigma_{i j}=\left(\sigma_{i}+\sigma_{j}\right) / 2$ and $\sigma_{i}$ is the radius of particle $i$. The compression then proceeds in a succession of small instantaneous particle inflation followed by energy minimization using conjugate gradient 2]. At large volume fraction, the energy cannot be minimized to zero anymore, and particles now overlap, signalling that the jamming transition has been crossed.

\begin{tabular}{|c|cccc|}
\hline$\phi_{\text {init }}$ & 0.3572 & 0.5397 & 0.5672 & 0.5935 \\
\hline$\phi_{J}\left(8000, \phi_{\text {init }}\right)$ & $\mathbf{0 . 6 4 8 1}$ & $\mathbf{0 . 6 4 9 9}$ & $\mathbf{0 . 6 5 3 7}$ & $\mathbf{0 . 6 6 1 6}$ \\
$\phi_{J}\left(1000, \phi_{\text {init }}\right)$ & 0.6466 & 0.6491 & 0.6531 & 0.6616 \\
\hline$\sigma_{J}\left(8000, \phi_{\text {init }}\right)$ & $5.2 \cdot 10^{-4}$ & $4.9 \cdot 10^{-4}$ & $3.0 \cdot 10^{-4}$ & $2.5 \cdot 10^{-4}$ \\
$\sigma_{J}\left(1000, \phi_{\text {init }}\right)$ & $9.7 \cdot 10^{-4}$ & $1.0 \cdot 10^{-3}$ & $7.6 \cdot 10^{-4}$ & $5.8 \cdot 10^{-4}$ \\
\hline$z$ at $\phi_{J}$ & 6.0017 & 6.0022 & 6.0021 & 6.0023 \\
$\sigma_{z}$ & 2.47 & 2.50 & 2.54 & 2.65 \\
$z_{12}$ at $\phi_{J}$ & 2.92 & 2.91 & 2.92 & 2.97 \\
\hline Rattlers & $4.3 \%$ & $4.7 \%$ & $5.4 \%$ & $6.2 \%$ \\
\hline
\end{tabular}

TABLE I: Statistics of jammed configurations for different system sizes $N$ and various $\phi_{\text {init }}$. The limit $\phi_{J}\left(N \rightarrow \infty, \phi_{\text {init }}\right)$ exists, with finite $N$ fluctuations decaying in good agreement with $N^{-1 / 2}$, and retains a dependence on $\phi_{\text {init. }}$. At the transition, configurations are all isostatic, $z \approx 6$ (with similar distribution width $\sigma_{z}$ ), nearly identical local structure, number of rattlers, and no increasing demixing between species ( $z_{12}$ is nearly constant).

We emphasize that all the details entering this compression protocol in principle quantitatively affect the results presented below 23]. However, since the most sensitive control parameter is $\phi_{\text {init }}$, we shall only use its value to distinguish between the different protocols, all other parameters being kept fixed. We focus on four values for $\phi_{\text {init }}$ listed in Table [ We monitor finite size effects and convergence towards the thermodynamic limit by studying two system sizes, $N=1000$ and 8000, chosen in the regime where scaling with $N$ is well understood [2].

In Fig. 1 1 a we show the final part (e.g. when particles are treated as soft) of a randomly selected compression history for a system with $N=10^{3}$. We follow the energy density of the system, $e=N^{-1} \sum_{i<j} V_{\text {soft }}\left(r_{i j}\right)$, for increasing $\phi$. We have defined configurations having energy density less than $10^{-16}$ as unjammed. For low enough $\phi$, we obtain only unjammed particle configurations. Upon increasing the density, there appears a volume fraction above which the energy increases rapidly above zero: this is the jamming transition [2]. Upon further compression, the energy keeps increasing on average, and so does the average number of contacts per particle, $z$.

This qualitative description becomes more complicated when multiple histories are superimposed, see Fig. 1 tb. There are several sources of fluctuations in this plot. A first source of fluctuations stems from the possibility for the system to undergo some reorganization which makes the energy drop suddenly, and makes the $e(\phi)$ curve multivalued during a single compression run. Thus, no simple scaling law for the energy can be detected during compressions. The second source of fluctuations arises when different realizations of the same protocol (i.e. same value of $\phi_{\text {init }}$ but starting from independent fluid configurations) are compared. These fluctuations are present because $N$ is finite, and should vanish when $N \rightarrow \infty$. A third source of fluctuations can be seen in Fig. 1] when 

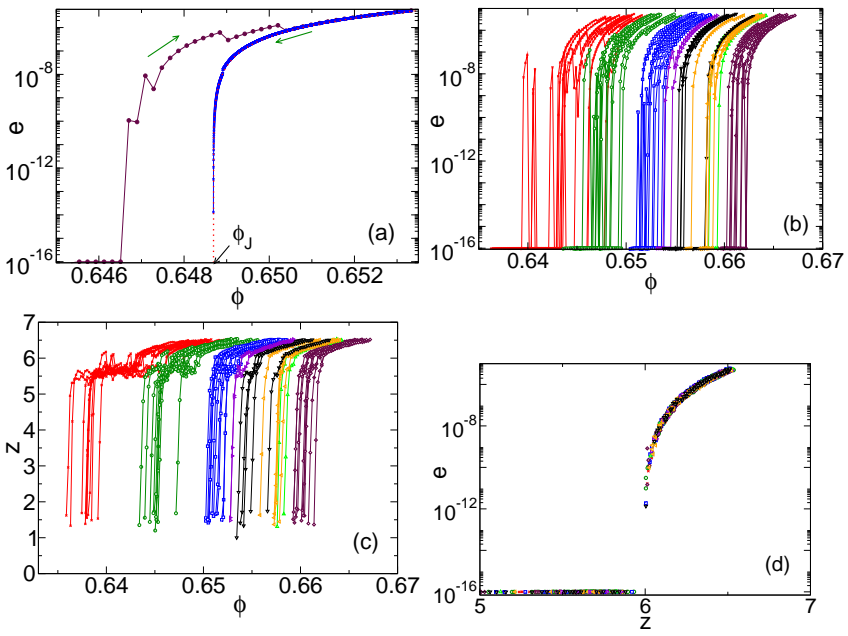

FIG. 1: (a) The final part of a compression-decompression cycle across the jamming transition for $N=10^{3}$ and $\phi_{\text {init }}=$ 0.3572 , showing the practical determination of $\phi_{J}$ for this sample. (b) Evolution of the energy density during compressions of several runs with $N=1000$. Different curves with the same color correspond to independent realizations at constant $\phi_{\text {init }}$, while different $\phi_{\text {init }}$ are shown with different colors. (c) Same as (b) for the evolution of the number of contacts per particle. (d) All data in (b) and (c) collapse when $\phi$ is eliminated, showing that the jamming transition is better defined by its properties than by its location.

$\phi_{\text {init }}$ is varied, larger $\phi_{\text {init }}$ typically yielding larger volume fraction for the onset of positive energies.

If we decompress jammed configurations, reorganizations are much less likely, and the $e(\phi)$ curve is smooth enough that it allows a quantitative determination of $\phi_{J}$, see Fig. 1.a. Of course, $\phi_{J}$ now depends on one additional parameter, namely the volume fraction from which decompression starts. We arbitrarily decompress when $e \approx 10^{-7}$ and obtain $\phi_{J}$ by fitting $e$ to a power law decay, $e \sim\left(\phi-\phi_{J}\right)^{2}$ during decompression. Thus, we determine $\phi_{J}=\phi_{J}\left(N, \phi_{\text {init }}\right)$. We repeat this analysis for different $N, \phi_{\text {init }}$, and initial configurations to obtain the statistics of $\phi_{J}$ reported in Table I. The main result of this analysis is that $\phi_{J}\left(N \rightarrow \infty, \phi_{\text {init }}\right)$ seems sharply defined (the fluctuations decrease in good agreement with the $N^{-1 / 2}$ scaling [2]) for each value of $\phi_{\text {init }}$, but the $\phi_{\text {init }}$ dependence survives a substantial increase of system size. Therefore, we have directly established that, for frictionless spheres, the jamming transition occurs along a continuous line rather than at a specific point in volume fraction, as hinted in earlier simulations [19, 22], and predicted theoretically [14, 15].

Having found several critical points for jamming, rather than one, we now ask whether these different $J$-points are equivalent. We have measured the static structure of the jammed configurations through the (partial) pair correlation functions $g_{\alpha \beta}(r)(\alpha, \beta=1,2$ for small/large particles, respectively) and the statistics of

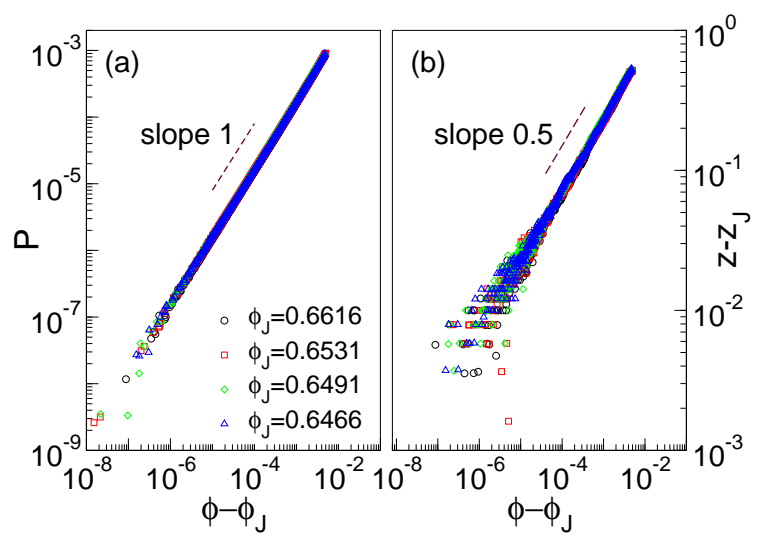

FIG. 2: Scaling behaviour along the continuous line of $J$ points. The pressure vanishes as $P \sim\left(\phi-\phi_{J}\right)$ (left axis) while the number of contacts (right axis) is discontinuous, $z \sim z_{c}+\sqrt{\phi-\phi_{J}}$ with $z_{c} \simeq 6$. We show data for 4 representative points $J$ with 4 different $\phi_{J}$.

contacts between particles. We have also followed the properties of configurations approaching jamming from large volume fraction. As in [2], we find several scaling laws near our four $J$-points, for $P, e$ and $z$. In Fig. 2 we show that for all $J$-points we have $P \sim\left(\phi-\phi_{J}\right)$ and $z=z_{c}+\sqrt{\phi-\phi_{J}}$ with $z_{c} \simeq 6$ (see Table $\llbracket$. Within our numerical precision, the critical properties of jamming transitions along the $J$-line are identical.

This analysis suggests that the value of $\phi_{J}$ is strongly influenced by the entire sample history (compression/decompression protocol, reorganization during compressions, etc.), but that the scaling properties of the jamming transition are much more robust. It is tempting to formulate the appealing hypothesis that the history dependence of the jammed configurations are contained solely in the volume fraction $\phi_{J}$ at which they jam. This would suggest that data analysis of the jamming transition should drastically simplify if we eliminate $\phi_{J}$ from consideration. We confirm the validity of our hypothesis in Fig. 1.d, where the three sources of fluctuations discussed above are accounted for by eliminating $\phi$ from the description. By plotting $e$ vs. $z$, we find that data for all cases nicely collapse onto a mastercurve, $e \sim\left(z-z_{c}\right)^{4}$, independently of the sample preparation history. Thus, the details of the numerical protocols are irrelevant to the relationship between energy and coordination number across the jamming transition.

Not only are scaling properties near jamming robust, but the special structure of the pair correlation function at the transition is also observed in all our samples. The pair correlation has a delta peak at contact, which, when integrated yields the average number of contacts, see Table I. The total number, $z$, and partial numbers, $z_{\alpha \beta}$, have the same value for all $J$-points, within numerical accuracy. Note in particular that $z_{12}$ does not evolve, indicating that demixing between species of the mixture 

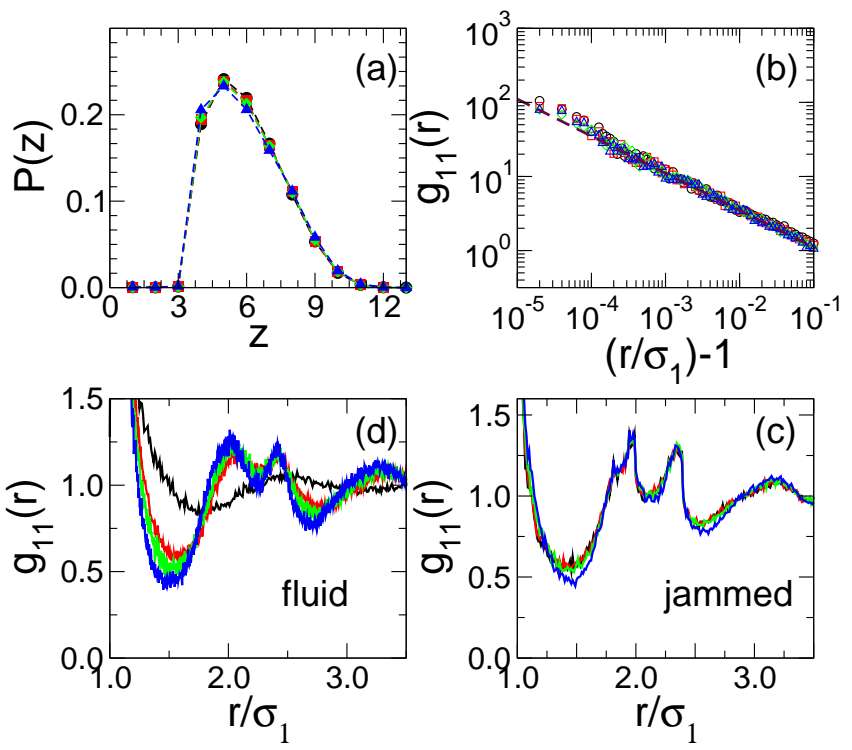

FIG. 3: (a) Statistical distribution of the number of contacts per particle, $z$, for 4 points $J$. (b) Power law singularity near contact for $g_{11}(r) \sim\left(r-\sigma_{11}\right)^{\gamma}$ for the four points $J$, with $\gamma=\frac{1}{2}$ shown as dashed line. (c) $g_{11}(r)$ near second and third peaks for 4 points $J$. (d) As in (c) for the equilibrated fluid configurations at $\phi_{\text {init }}$.

is not observed. In fact the full distributions of contact number barely change, see Fig. 3-a. The pair correlation also has a power law singularity near contact [8, 9], $g_{\alpha \beta}(r) \sim\left(r-\sigma_{\alpha \beta}\right)^{\gamma}$. This is shown in Fig. 3 b for $g_{11}(r)$, where again the exponent is the same for the four $J$ points, $\gamma \approx 0.5$. In Fig. 3 3 c, we observe a subtle but systematic evolution with $\phi_{\text {init }}$ of $g(r)$ from near the first minimum to the third peak. Similar to the case of inherent structures in systems with soft potentials, these subtle variations reflect the more clearly visible changes in the structure of the equilibrated fluid configurations at $\phi_{\text {init }}$ which were used as starting points for the compressions, as shown in Fig. 3-d. Finally, we note that the increase of $\phi_{J}$ does not result simply from a decreasing number of rattlers (particles with no contact), see Table I.

Our results directly establish that a unique location for the jamming transition cannot be expected, even when crystallization or demixing play no role. Rather, our findings are broadly consistent with a 'landscape' picture wherein the fluid explores a phase space that does not form the basin of a well-defined, unique ground state, but instead one marked by the presence of many local minima or metastable states, as is typical of glassy systems. The basins predominantly sampled vary with the equilibrium volume fraction of the system. As in thermal systems 24] using the inherent structure formalism, the nature, number and evolution with volume fraction of these metastable states can be evaluated 20], and are described exactly in the mean-field limit [14, 15]. An eval- uation of configurational entropy associated with these jammed states and a comparison with the sharp boundaries predicted in mean-field theory, corresponding to the appearance and vanishing of a configurational entropy, would be valuable for this system.

In summary, we have shown that jamming transitions of amorphous packings of frictionless spheres occur along a continuous range of volume fraction. These transition are sharply defined in the thermodynamic limit, and this finite range exists even when ordering phenomena or friction are absent, contrasting with the idea of a unique jamming point. Since the location of the jamming transition in fact results from the specific protocol used to study it, we see no reason why it should be reproducible from one experiment to another.

We would like to thank J.-L. Barrat and J. Kurchan for useful exchanges. PC acknowledges financial support from UCBL1 and ANR SYSCOMM.

[1] J. D. Bernal and J. Mason, Nature 188, 910 (1960).

[2] C. S. O'Hern, S. A. Langer, A. J. Liu, and S. R. Nagel, Phys. Rev. Lett. 88075507 (2002).

[3] C. Song, P. Wang, and H. A. Makse, Nature 453, 629 (2008).

[4] C. S. O'Hern, S. A. Langer, A. J. Liu, and S. R. Nagel, Phys. Rev. E 68, 011306 (2003).

[5] C. Heussinger and J.-L. Barrat, Phys. Rev. Lett. 102, 218303 (2009).

[6] P. Olsson and S. Teitel, Phys. Rev. Lett. 99, 178001 (2007).

[7] T. Hatano, J. Phys. Soc. Jpn. 77, 123002 (2008).

[8] A. Donev, S. Torquato, and F. H. Stillinger, Phys. Rev. E 71, 011105 (2005).

[9] L. E. Silbert, A. J. Liu, and S. R. Nagel, Phys. Rev. E 73, 041304 (2006).

[10] A. J. Liu and S. R. Nagel, Nature 396, 21 (1998).

[11] S. Torquato, T. M. Truskett, and P. G. Debenedetti, Phys. Rev. Lett. 84, 2064 (2000).

[12] G. Brambilla, D. El Masri, M. Pierno, G. Petekidis, A. B. Schofield, L. Berthier, and L. Cipelletti, Phys. Rev. Lett. 102, 085703 (2009).

[13] S. Sastry, P. G. Debenedetti and F. H. Stillinger, Nature 393, 554 (1998).

[14] F. Krzakala, and J. Kurchan, Phys. Rev. E 76, 021122 (2007).

[15] G. Parisi and F. Zamponi, Rev. Mod. Phys. (in press); arXiv:0802.2180

[16] R. J. Speedy, J. Phys: Condens. Matter 10, 4185 (1998).

[17] R. J. Speedy and P. G. Debenedetti, Mol. Phys. 88, 1293 (1996).

[18] A. Donev, F. H. Stillinger, and S. Torquato, J. Chem. Phys. 127, 124509 (2007).

[19] M. Hermes and M. Dijkstra, arXiv:0903.4075.

[20] R. J. Speedy, J. Chem. Phys. 100, 6684 (1998).

[21] Y. Brumer and D. R. Reichman, Phys. Rev. E 69, 041202 (2004).

[22] L. Berthier and T. A. Witten, Phys. Rev. E 80021502 (2009). 
[23] A. Donev, S. Torquato, F. H. Stillinger, and R. Connelly, Phys. Rev. E 70, 043301 (2004).

83, 3214 (1999).

[24] F. Sciortino, W. Kob and P. Tartaglia, Phys. Rev. Lett. 